\title{
Scalar Theory of Everything (STOE) unites the big, the small, and the four forces (GUT) by extending Newton's model
}

\author{
John C. Hodge \\ Retired, 477 Mincey Rd., Franklin, NC, 28734 \\ E-mail: jchodge@frontier.com
}

\begin{abstract}
Newton established a major physics advance that was confirmed by predicting the late return of Halley's comet. Newton identified three characteristics of bodies that have been identified as three characteristics of "mass". Current standard physical models have become very complex with divergent postulates for cosmology and quantum mechanics. Physics theory restarting from Newton's speculations and then describing the experiments of the 19th and 20th centuries results in a model of the big, the small, and the four forces (GUT) - the Scalar Theory of Everything (STOE).
\end{abstract}

keywords: TOE, GUT, Newton, mass

\section{INTRODUCTION}

Human physical models must begin with some postulates, evolve to explain observations, and make useful predictions. Newton created a theory of gravity with his three laws of motion [1]. This model related the motion of bodies on Earth and of the Moon. The key measurable characteristics of bodies were assumed. Later, Newton speculated on the common postulates for gravity and light - the big and the small. The predicted late return of Halley's comet confirmed Newton's model for the solar system scale. Thus, astronomical happenings were related to Earth-born experiments. A key characteristic of Newton's model is that it is simpler and describes a wider range of observations than the other models it replaced.

Newton identified three characteristics of bodies that are different views of mass characteristics. This is the postulates stage. Definitions become better defined as they are related to observations. Weight $\left(m_{\mathrm{w}}\right)$ was defined as a body's density times its volume and as the "quantity of matter" in a body. A body's density, volume, and innate force of matter are our everyday understood parameters. Inertial mass $\left(m_{\mathrm{i}}\right)$ was defined as a body's innate force of matter with 


\section{INTRODUCTION}

the power of resisting a change in its state of motion. This force is exercised only when another force impressed on the body changes the body's motion. Gravitational mass $\left(m_{\mathrm{g}}\right)$ was presented in his third book of the Principia. It is the body's characteristic that instantaneously impresses an attractive force on another body over a distance in absolute space. The electromagnetic forces were unaccounted in Newton's time. Fluid and other forces were outside Principia. Newton recognized that a gravity only model created a problem in cosmology requiring an infinite, eternal universe and requiring an infinite force as distances between centers of mass approached zero. The motion of the moon and Sun suggested instantaneous reaction to the force of gravity. A more complete description may be found in Assis [2, Section I and III].

Newton's model works by defining (mapping) forces that are exerted on matter then adding force vectors to arrive at a single force that predicts the mass's measurable motion by inverse mapping. This requires the mathematical constants of proportionality to be such that the body's motion is as observed.

Later, Newton expanded on how $m_{\mathrm{g}}$ may be impressed on another body and the relation of gravity and light, stated in modern terms [3]:

Qu. 1 Masses bend passing light by an amount inverse to distance.

Qu. 3 Light passing close to edges are diffracted in fringes.

Qu. 4 Light path begins to bend BEFORE reaching a body or slit.

Qu. 5 Light heats mass.

Qu. 6 Black bodies absorb all light.

Qu. 8 Black body radiation.

Qu. 11 Sun and stars are black body radiators.

Qu. 17 Light is corpuscles that are pushed around (divergence of the aether's density) by waves of the aether that "overtake" the rays of light. The waves in the aether travel faster than light - its not a big stretch to say MUCH faster than light. That is, gravity is not force. Gravity is the effect of the divergence of the aether, also.

Qu. 18 A Medium that refracts and reflects light also allows light to heat bodies. Newton also suggested light travels faster in the void (denser parts of the aether). This is the measured Shapiro delay.

Qu. 19 Refraction of light implies differing densities of the Medium (calls it "...this aether Medium..."). Density of the aether is GREATER in "...free and open spaces void of air and other grosser bodies...". How the aether became denser between bodies was unexplained.

Qu. 20 Increasing aether density (ie divergence) causes light refraction. 
Qu. 21 Aether rarer within dense bodies and increase with distance and "...thereby cause the gravity of those great bodies; every body endeavoring to go from the denser parts of the medium towards the rarer?". He also suggested the aether is so rare as to not impede the planets revolution (no aether wind).

Newton suggested a reductionism step by positing (1) an aether whose divergence exerted a force that caused gravity and that caused the diffraction, reflection, and refraction of light and (2) corpuscles as light and as components of matter. That is, support for the aether and corpuscles was because the model could explain several diverse observations then known but mysterious rather than by direct observation.

This Paper suggests the Scalar Theory of Everything (STOE) is a continuation of Newton's model. Section 2 briefly describes the deviation from Newton's path and some observations after Newton's time. Section 3 describes a STOE model of the three types of mass as independent physical effects. The Discussion and Conclusion are in section 4.

\section{THE DEVIATION FROM NEWTON'S PATH}

Young's Experiment of interference was interpreted to indicate light was a wave rather than corpuscles and rather than the wave interference was caused by the aether (Newton used a rock-thrown-into-water analogy). This began the effort to attempt to explain observations of the small scale by waves, fields, etc. This effort has resulted in the very counterintuitive (no analogies with the classical world) Quantum Mechanics and the loss of the connection to gravity. This has resulted in the incompatibility of General Relativity and Quantum Mechanics.

Several experiments in electricity and magnetism were combined into Maxwell's Equations rather than discovering how Newton's three masses and a single, continuous aether could explain the experiments. Because the experiments were independently done, several simplifications resulted in incorrect descriptions $[4 ; 5 ; 6 ; 7]$. The electromagnetic signals have a speed of light. If light is a wave, then the speed of a wave is the speed of light. However, this has been falsified for the speed of coulomb fields [8].

The invention of the Hamiltonian and the Lagrangian that calculated a body's motion in terms of energy resulted in the distinction of the three masses being lost. The $m_{\mathrm{w}}$ had been considered as an $m_{\mathrm{i}}$ and these functions considered the distinction between kinetic energy and potential energy as caused by one type of mass. However, Newton argued the $m_{\mathrm{i}}$ and $m_{\mathrm{g}}$ were distinct but proportional which were included in the gravitational constant $G$. Models currently exist that suggest the $G$ varies across a galaxy such as MOND.

Mach's Principle, which is vague, suggested that the stars in the universe should influence measurements (inertia) on Earth. Mach's Principle is viewed as suggesting a total relativism and as a substitute for Newton's "absolute space". However, because the influence is by bodies, the higher aether density required by Newton (so the $m_{\mathrm{g}}$ can reduce the aether) is unaccounted. 


\section{THE STOE}

The well-known Einstein thought experiment of a measuring system in a (small) box (such as an elevator) being unable to determine if the box is in a gravity well or under acceleration of a rocket resulted in the Strong Equivalence Principle. But this is incorrect. Gravity acts as if from a point (center of mass). Therefore, gravity shows the force vectors as converging. The rocket shows force vectors as parallel. The "small" condition to achieve equality is more properly a measurement uncertainty. The "small" condition was ignored and the equality of acceleration and gravity was accepted.

The left side of the General Relativity Field equation (LHS) is a mathematical mapping of observed energy and momentum into $s$ and $t$ parameters called "spacetime". But like Newton's aether it has a high value where no mass exists such as between galaxies and depressed values around masses. From whence, this "spacetime"? That is, the "spacetime" (LHS) cannot be real. Therefore, it is a mapping that must be inverse-transformed to real energy and momentum measurements.

General Relativity and other metric theories are NOT Machian models although there have been several attempts.

Because the stars of galaxies are modeled as the source of the Machian influence, the background of all "spacetime" must decrease (like Newton's model). The Big Bang model suggests a high amount of the stuff of the universe between galaxies that infalls into galaxies. However, observations falsify the infall model for spiral galaxies. Rather, spiral galaxies eject matter. However, elliptical galaxies do have infall characteristics [9].

The discovery of phenomena suggesting an atomic structure resulted in attempting to describe the spectra as an orbital (angular momentum) characteristic of electrons [10]. This became untenable because radiation should be emitted and none was observed for stable structures. Later attempts became weirder and more complex by using Quantum Mechanics combined with many ad hoc exclusion rules.

Many observations suggest several parameters are "fine-tuned" such as the temperature of the microwave background. Fine-tuning remains unexplained.

The increase number and accuracy of observations has resulted in physical models becoming more complex and separated into at least 3 major incompatible disciplines - Relativity, Quantum mechanics, and subatomic particles (group theory) including the Grand Unified Theory (GUT).

\section{THE STOE}

The return to Newton's path started with the Quasi Steady State Cosmology (QSSC) [11; 12]. QSSC suggested a Source of the stuff of our universe at the center of galaxies. The rotation curves of spiral galaxies were explained as a medium density directing bodies to vary as $1 / d$ ( $d$ being the distance from the Source) according to the spherical principle. Note the positive value that causes an outward force on bodies. Matter moving outward combines to form heavier bodies that are gravitationally attracted inward in spiral galaxies. However, 


\section{THE STOE}

some matter escapes as the $1 / d$ pushes it beyond the galaxy's gravitational influence.

For the volume between galaxies to have low medium density, the universe must expand. Because the Sources adds the stuff of the universe, the QSSC is Machian. However, the QSSC fails when describing elliptical galaxy characteristics.

The STOE posits the components of the universe are discrete hods and a continuous plenum that is a medium that supports waves similar to Newton's aether. Because the plenum supports waves, it has the property of inertia [13; 14], has plenum density $\rho$, it directs hods, and it has waves with a speed much greater than light.

Hods are 2 dimensional, magnetic with a high $\rho$ on one side and $\rho=0$ on the other, and particles are assemblies of hods $[15 ; 16]$. Hods travel at the speed of light in the direction parallel to their surface and slower in a direction perpendicular to their surface.

The STOE adds Sinks to the QSSC that are elliptical galaxies in galaxy clusters that lowers the $\rho[17 ; 18 ; 19]$. The stuff of the universe flows into the Sinks which explains the many differences between spiral and elliptical galaxies [9]. The Sinks remove the need for an expansion of the universe and for infall into spiral galaxies.

The universe begins as a single Source that eject other Sources [19; 20]. The entire universe emerges (strong emergence) from the two components and their interaction [21].

The STOE suggested a Universal Equation $[22 ; 23 ; 24 ; 25]$. The plenum density at all points $\rho_{\mathrm{p}}$ in the universe is the sum of the effects of all galaxies and all hods,

$$
\begin{aligned}
\rho_{\mathrm{p}}= & K_{\epsilon} \sum_{i=1}^{N_{\text {source }}} \frac{\epsilon_{i}}{r_{i}}-K_{\eta} \sum_{j=1}^{N_{\text {sink }}} \frac{\eta_{j}}{r_{j}} \\
& \quad-K_{\text {hods }} \sum_{k=1}^{N_{\text {hods }}} \frac{m_{\mathrm{g}}}{r_{k}} \cos \left(\frac{2 \pi r_{k}}{\lambda_{\mathrm{T}}}-\pi\right) \exp ^{-j\left(\omega t_{k}\right)} \\
>0 & ,
\end{aligned}
$$

where $K_{\epsilon}, K_{\eta}$, and $K_{\text {hods }}$ are constants that relate the relative influence of the Sources, Sinks, and hods, respectively; $\epsilon_{i}, \eta_{j}$, and $m_{\mathrm{g}}$ are constants that relate the value of a measurable parameter such as a galaxy's B-band luminosity or hod gravitational effect for Sources, Sinks, and hods, respectively; $r_{i}, r_{j}$, and $r_{k}$ are distances from the center of each component to the point being evaluated; $\lambda_{\mathrm{T}}$ and $t_{k}$ are wave characteristics generated in the plenum by moving hods. The $\lambda_{\mathrm{T}}$ and $t_{k}$ depend on the $\rho_{\mathrm{p}}[26]$.

The $\rho$ as the $r$ increases, like a fluid, becomes smoothed in the direction perpendicular to $r$. This is the "spherical principle". The number of hods appear as if they were concentrated at the center of the structure (center of mass) as $r$ increases beyond a minimum value. The $K_{\text {hods }}$ term looks like the gravitational mass effect in Newtonian scale observations. 


\section{THE STOE} tions.

The $K_{\epsilon}$ and $K_{\eta}$ terms become predominate on cosmological scale observa-

The inclusion of all Sources, Sinks, and matter means the STOE is a Machian model.

Note the $r$ values are for the determination of distance for the $\rho$ calculation. Therefore, the time required for a change in $r$ to travel through the plenum determines the position of the emitting object for the calculation of $\rho$ at a point. The change-in- $r$ wave can be very much faster than the speed of light $[27]$.

The divergence of $\rho_{\mathrm{p}}$ is proportional to a force $\vec{F}_{\mathrm{s}}$ that acts on matter and directs a hod,

$$
\vec{F}_{\mathrm{s}}=K_{\mathrm{G}} \sum_{l=1}^{N_{\mathrm{hods} l}} m_{\mathrm{hod}}\left(\vec{n}_{l} \bullet \vec{\nabla} \rho_{\mathrm{p} l}\right) \vec{n}_{l},
$$

where $m_{\text {hod }}$ is the surface area of a hod that is the same for all hods and $\vec{n}_{l}$ is the unit vector perpendicular and centered on the hod's surface. .

For matter,

$$
\vec{F}_{\mathrm{s}}=G_{\mathrm{s}} m_{\mathrm{w}} \vec{\nabla} \rho_{\mathrm{p}}
$$

where the $G_{\mathrm{S}}$ is a proportionality constant analogous to the gravitational constant $G$ without the $m_{\mathrm{i}} / m_{\mathrm{g}}$ value. The $m_{\mathrm{w}}$ is experienced as the effective surface area of the particle, and the familiar negative sign is included in the $\vec{\nabla} \rho_{\mathrm{p}}$.

Because electromagnetic signals speed is that of light, electromagnetic signals are hods [5]. The amount of plenum captured by a hod is constant and is the cause of $m_{\mathrm{i}}$ of bodies. Photons are a column of hods [26]. Because hods are two dimensional, hods and photons experience no impressed force in their direction of travel. That is, for smaller than electron structures such as photons or neutrinos, there can be directions with varying force depending on direction and $m_{\mathrm{w}}=0$ in the direction of travel. Therefore, they travel at the maximum allowed speed of other bodies.

The structure of baryonic matter has hod surfaces facing all three directions such that the effective surface area is less than the number of hods times $m_{\text {hod }}$ $[15]$.

The inverse mapping produces the movement of a body:

$$
\vec{a}=\frac{\vec{F}_{\mathrm{s}}}{m_{\mathrm{i}}}
$$

where $m_{\mathrm{i}} \neq 0$ because it is the captured plenum around the hods in the body and $\vec{a}$ is the acceleration of the body. Reference frames result when some terms of Eq. 1 are ignored for calculation simplicity.

Newton's three masses are (1) $m_{\mathrm{w}}$ is the effective surface area on which the $\vec{F}_{\text {s }}$ is exerted, (2) $m_{\mathrm{i}}$ is the captured plenum which is proportional to the number of hods in a body, and (3) $m_{\mathrm{g}}$ is the deformation of the plenum caused by the hods and is proportional to the number hods in a body. Note the number of effective hods vary by type of assembly (photon, neutrino, or baryon [15]). 
The Universal Equation has quantitatively described many mysterious observations $[23 ; 25 ; 28]$ :

Microwave background temperature is due to a fine-tuning due to a feedback loop between Sources and Sinks [29]. This suggests that if fine-tuning is evident, then a physical feedback loop is present.

Redshift $(z)$ was calculated by considering each galaxy's effect on the photon [30]. That is, the $\rho_{\mathrm{p}}$ is calculated for points along the photon's path (line of sight) using Eq. 1. The correlation coefficient of model distance versus Cepheid distance for the best Doppler shift model is 0.80. The STOE model correlation coefficient for the same galaxies is 0.88 . The "Hubble Law" was recovered without invoking a Doppler shift.

Periodic redshift was calculated by considering the Sink's effect (the $K_{\eta}$ term) on redshift [30]. If a Sink is located between the target and the Earth, the $z$ is increased. If a Sink is located beyond the target from the Earth, the $z$ is decreased. A galaxy cluster is a shell of Source galaxies around a core of Sink galaxies [9]. Therefore, galaxy clusters appear to be elongated along the line-of-sight from Earth. This phenomenon is called "the fingers-of-God" pointing toward Earth.

Rotation curves (RCs) were calculated by considering the Source strength. The Source strength is the $\epsilon$ term minus the $m_{\mathrm{g}}$ term for the mass internal to the radius in Eq. 1. The advantage is that all types of RCs are described in addition to standard models that explain only flat RCs [31;32;33].

Asymmetric RCs, which are unexplained in standard models, were calculated by considering the galaxy's' strength and a neighbor galaxy's strength and position [31; 32;33].

Correlation of central massive object mass and central velocity dispersion was explained [34].

Pioneer Anomaly of all 10 characteristics (not just the general value of the blueshift) were calculated by considering solar system matter (hods) and the Milky Way Source [35]. The equation used derived from the equation for galaxy redshift. That is, the $\rho_{\mathrm{p}}$ is calculated for points along the signal's path (line of sight) using Eq. 1. For example, the annual periodicity is explained by the Sun's relative position to the signal's path, which is mathematically similar to the observed periodic redshift . Because the same equation as the redshift equation was used, the similarity and sign difference to the Hubble constant is explained.

Predictions made in 2006 about the Pioneer Anomaly were confirmed in 2013 [36].

Photon diffraction and interference intensity patterns of both Young's Experiment and experiments that reject wave models of light were duplicated using a toy model computer simulation $[26 ; 37 ; 38 ; 39 ; 40 ; 41 ; 42 ; 43]$. 
Particle structure models and how the structures differ relative to photons that determine speed were created [15].

Modifications to Maxwell's Equations that better agree with experiment were described. The Biot-Savart Law was restated based on experiment $[4 ; 5 ; 6 ; 44]$. There are two different types of magnetic field, each with the same symbol $(\vec{B})[7]$.

The "Planet 9" observations and spiral galaxy rotation curves are due to the Source effect at the center of the Milky Way [45]. The effect was predicted in 2006 when examining the Pioneer Anomaly by noting the orbit of Pluto and Neptune. This effect may also influence the perihelion advance of Mercury [46].

The "ether wind" measurements are explained as the divergence of the plenum rather than a "wind" [47]. The "wind" model failed because the indicated direction did not match any possible Earth celestial movement direction.

The STOE model of the hod as a permanent magnet qualitatively suggested how hods form particles, the nature of particles, and the four forces of the Grand Unified Theory (GUT).

Magnetism in the STOE model is postulated to emerge from the hods and is a gradient of the plenum. Magnetism is observed in small subatomic particles and light. Therefore, the hods are magnets [5]. The application of Eqs: 1 and 2 suggests the $\vec{\nabla} \rho$ follows the magnetic lines of force seen in iron filings at the macro scale. The observation of two types of magnetic fields [7] imply the hod is either rotating charges or is a permanent magnet. Because rotating charges would emit radiation energy, the rotating charge model is rejected in the STOE. Therefore, the hod is a permanent magnetic. The self-similar (fractal) postulate suggests disk permanent magnets are a macro object that is analogous to hods.

Repulsion was measured between 2 columns of disk permanent magnets. The measurements showed an asymmetry between South poles repelling and North poles repelling [48]. Further, the repulsion force along the axis was inverse-distance when the magnets were close, inverse distance-cubed a bit further, and inverse distance-squared still further apart. The force perpendicular and centered on the axis was attractive and inverse-cubed of the distance from the dipoles.

Particles' magnetic fields were studied [49] for structures suggested previously identified (photons, neutrinos, electrons).

Electromagnets did not show the asymmetry that the permanent magnets demonstrated [50].

Atomic structure in the standard model is orbiting electrons. The fundamental problem is that rotating charges should radiate and have a declining 
orbital radius. This is not observed. Also observed is that changing energy radiation is photons. Photons in the STOE are columns of hods. So, the STOE atomic structure is of rods of photons determining and magnetically holding electrons in position around the nucleus [51].

Gravity emerges from magnetic effects in hod structures [52]. However, magnetic attractive and repulsive effects determine atomic structures. Therefore the distance required before an only attractive force is at least the atomic scale of Angstroms. That is, the "gravity" at more than atomic scale is an approximation which becomes invalid at less than atomic scale rather than being present and merely too small. The distance at which this occurs may determine atomic size and may suggest instability in larger atomic (dominated by inverse square distances) and nuclear (dominated by inverse cube distances) size. The relative strength of the photon hod binding and the gravity speaks to the "hierarchy problem". The Newtonian problem of $r \rightarrow 0$ for gravity causing an infinite force is nonexistent.

Nuclear forces (weak and strong) are modeled as an assembly of hods into a spherical ball which is the proton and neutron [53].

Grand Unified Theory is, therefore, that the magnetic force evolves with distance into the four forces of the GUT.

\section{DISCUSSION AND CONCLUSION}

Like Newton, the STOE suggests a reductionism step by positing the characteristics that can explain many problematic, mysterious observations.

The intent of $\vec{F}_{\mathrm{s}}$ is that it be all inclusive. The development of the STOE to date has demonstrated the interference effects and gravity. The hod is a magnet so the possibility is that electromagnetic effects may be modeled as approximations as was gravity. However, the equation and effects for very small values of the $r$ 's in Eq. 1 are lacking. Therefore this is an area for continued experimental research. Perhaps the STOE could be developed to model atomic and nuclear decay and to improve the model of galaxy redshift by including QSOs as H. Arp suggests [20].

Physics theory restarting from Newton's speculations and then describing the experiments of the 19th and 20th centuries results in a model of the big, small, and the four forces (GUT) - the Scalar Theory of Everything (STOE).

\section{ORCID iD}

John C. Hodge: https://orcid.org/0000-0002-1520-2153 


\section{REFERENCES}

\section{References}

[1] Newton, I., 1999. The Principia Mathematical Principles of Natural Philosophy A new translation by I. Bernard Cohen and Anne Whitman (University of California Press, Berkley, CA., USA).

[2] Assis, A.K.T., 2014 Relational Mechanics and implementation of Mach's Principle with Weber's Gravitational Force (C. Roy Keys Inc., Montreal, Quebec, Canada)

[3] Newton, I., 1952. Opticks based on the 1730 edition (Dover Publications, Inc., New York).

[4] Hodge, J.C. 2018c. Experiment supports STOE model and rejects the traditional model of a coulomb field. IntellectualArchive, v.7(2), 11-14. http://intellectualarchive.com/?link=item\&id=1927.

[5] Hodge, J. C. 2018d. Magnetostatics relation to gravity with experiment that rejects Biot-Savart Law, IntellectualArchive v.7(3), P. 1-8, 2018. http://intellectualarchive.com/?link=item\&id=1945.

[6] Hodge, J. C. 2018e. Another experiment rejects Ampere's Law and supports the STOE model, IntellectualArchive v.7(4), P. 6-10. http://intellectualarchive.com/?link=item\&id=1956.

[7] Hodge, J. C. 2018f. Two different types of magnetic field. IntellectualArchive, v. 7 (4), P. 1-5. http://intellectualarchive.com/?link=item\&id=1964.

[8] de Sangro, R. et al., 2012, Measuring Propagation Speed of Coulomb Fields https://arxiv.org/abs/1211.2913

[9] Binney, J., and Merrifield, M., 1998, Galactic Astronomy Princeton, NJ: Princeton University Press.

[10] Hertzberg, G., 1944, atomic spectra and atomic structure, (ISBN 13: 9789-486-60115-1, (Dover Publications, Inc., New York, New York, USA).

[11] Hoyle,F., Burbidge, G., \& Narklikar, J.V., 2000, A different approach to cosmology, (ISBN 9781469987361, Cambridge Univ. Press, Cambridge).

[12] Narklikar, J.V. et al. 2015. Gravitational wave background in the QuasiSteady State Cosmology, Mon. Not. Astron. Soc. V.451(2), p. 1390-1395. preprint http://arxiv.org/abs/1505.05494.

[13] Hodge, J. C., 2016b. Inertia according to the STOE, http://intellectualarchive.com/?link=item\&id $=1676$.

[14] Hodge, J. C., 2017a. STOE inertia, IntellectualArchive, v.6(1), P. 158,http://intellectualarchive.com/?link=item\&id=1814. 


\section{REFERENCES}

[15] Hodge, J. C. 2016c. Structure and spin of the neutrino, electron, and positron. IntellectualArchive, v. $5,(2), \quad 1-8$. http://intellectualarchive.com/?link=item\&id=1694.

[16] Hodge, J. C. 2016f. STOE model of the electron spin 1/2 observation. IntellectualArchive, $\quad$ v. $5(4), \quad 1-7$. http://intellectualarchive.com/?link=item\&id=1735.

[17] Hodge, J. C. 2019a. QSSC model's next step is the STOE. IntellectualArchive, v. 8 (1), P. 9. http://intellectualarchive.com/?link=item\&id=2043.

[18] Hodge, J. C. 2019b. STOE beginnings. IntellectualArchive, v. 8(2), P. 11. http://intellectualarchive.com/?link=item\&id=2087.

[19] Hodge, J. C. 2020b. Nature of beginning. IntellectualArchive, v. 9 (2), P. 8. http://intellectualarchive.com/?link=item\&id=2343.

[20] Arp, H., 1998, Seeing Red: Redshifts, Cosmology and Academic Science Montreal, Quebc, Canada: Apeiron.

[21] Hodge, J. C., 2016g. STOE emergence, IntellectualArchive, v.5 (5), P. 1014, http://intellectualarchive.com/?link=item\&id $=1757$.

[22] Hodge, J. C. 2018g. Scalar Theory of Everything replacement of Special Relativity. IntellectualArchive, v.7(5), P. 1-8. http://intellectualarchive.com/?link=item\&id=1974.

[23] Hodge, J. C. 2012b. Theory of Everything: Scalar Potential Model of the big and the small(ISBN 9781469987361, available through Amazon.com).

[24] Hodge, J.C., 2018h, STOE replaces relativity and quantum mechanics, (ISBN 978-613-9-91465-4, (LAP LAMBERT Acedemic publishing, Mauritius, available through Amazon.com).

[25] Hodge, J. C., 2018i, STOE replaces relativity and quantum mechanics, IntellectualArchive, v.7(5), P. 98,. http://intellectualarchive.com/?link=item\&id=1979

[26] Hodge, J. C. 2012a. Photon diffraction and interference. IntellectualArchive, v. 1 (3), 31-60. http://intellectualarchive.com/?link=item\&id=597

[27] von Flandern, T., 1998. The speed of gravity-what the experiments say, Physics Letters A 250, 1.

[28] Hodge, J. C., 2010 Scalar potential model of galaxies: review and new speculations. Chapter 14 of Black Holes and Galaxy Formation, eds. A. D. Wachter and R. J. Propst, Nova Science Publishers, Inc (New York, USA).

[29] Hodge, J. C. 2006a. Scalar potential model of the CMB radiation temperature. https://www.arxiv.org/abs/astro-ph/0603140. 


\section{REFERENCES}

[30] Hodge, J. C. 2006b. Scalar potential model of redshift and discrete redshift. NewA, v.11 (5), 344-358. http://www.arxiv.org/PS_cache/astro$\mathrm{ph} / \mathrm{pdf} / 0602 / 0602344 \mathrm{v} 1$.pdf.

[31] Hodge, J. C. 2003a. Relationship Between Cepheid and Tully-Fisher distance Calculations. Presented at 202nd meeting of the AAS, session 40.08. https://www.arxiv.org/abs/astro-ph/0304030.

[32] Hodge, J. C. 2003b. Neighboring Galaxies' Influence on Rotation Curve Asymmetry. Presented at 202nd meeting of the AAS, session 40.08. https://www.arxiv.org/abs/astro-ph/0305022.

[33] Hodge, J. C. 2006c. Scalar potential model of spiral galaxy HI rotation curves and rotation curve asymmetry. http://www.arxiv.org/PS_cache/astro$\mathrm{ph} / \mathrm{pdf} / 0611 / 0611029 \mathrm{v} 2$.pdf.

[34] Hodge, J. C. 2006d. Scalar potential model of galaxy central mass and central velocity dispersion. http://www.arxiv.org/PS_cache/astro$\mathrm{ph} / \mathrm{pdf} / 0611 / 0611699 \mathrm{v} 1 . \mathrm{pdf}$

[35] Hodge, J. C. 2006e. Scalar potential model of the Pioneer Anomaly. http://www.arxiv.org/PS_cache/astro-ph/pdf/0602/0612567v1.pdf.

[36] Hodge, J. C. 2013a. Pioneer Anomaly predictions confirmed. http://intellectualarchive.com/?link=item\&id=1088.

[37] Hodge, J. C. 2015a. Single Photon diffraction and interference. IntellectualArchive, v. 4 (4), 1-11. http://intellectualarchive.com/?link=item\&id=1557

[38] Hodge, J. C. 2015b. Diffraction experiment and its STOE photon simulation program rejects wave models of light. IntellectualArchive, v. 4(6), 6-16. http://intellectualarchive.com/?link=item\&id=1603.

[39] Hodge, J.C., 2016d. Hodge Experiment distinguishes between wave and particle caused diffraction patterns, IntellectualArchive, v. $5(3)$, P. $7==10$, http://intellectualarchive.com/?link=item\&id=1712.

[40] Hodge, J. C., 2016e. STOE assumptions that model particle diffraction and that replaces QM, IntellectualArchive, v.5(3), P. 1-6, http://intellectualarchive.com/?link=item\&id=1719.

[41] Hodge, J. C., 2017d. Hodge experiment (continued) with opaque strips and about the Afshar Experiment, IntellectualArchive, v.6(5), P. 7-18, http://intellectualarchive.com/?link=item\&id=1872.

[42] Hodge, J. C., 2017e. STOE simulation of photon spectrographic behavior, IntellectualArchive, v.6(6), P. 1-6, http://intellectualarchive.com/?link=item\&id=1884. 


\section{REFERENCES}

[43] Hodge, J. C. 2019e. Interference Experiment with a transparent Mask Rejects Wave Models of Light, Optics and Photonics Journal, v.9(6). DOI: 104236/opj.2019.96008. http://www.scrip.org/journal/paperinformation.aspx?paperid=93056.

See video: https://www.youtube.com/watch?v=A07bogzzMEI

[44] Hodge, J. C. 2018b. STOE electric charge. IntellectualArchive, v. 7 (2), 110. http://intellectualarchive.com/?link=item\&id=1917.

[45] Hodge, J. C. 2019c. STOE explains "Planet 9" . IntellectualArchive, v. 8 (2), P. 13. http://intellectualarchive.com/?link=item\&id=2089.

[46] Hodge, J. C. 2020a. Sgr.A* effect on Mercury and Trans-Neptune Objects. IntellectualArchive, v.9(1), P. 11. http://intellectualarchive.com/?link=item\&id=2266.

[47] Hodge, J. C. 2019d. STOE explaination for the "ether wind". IntellectualArchive, v.8(2), P. 15. http://intellectualarchive.com/?link=item\&id=2111.

[48] Hodge, J. C. 2019f. Magnetic field evolves to gravity field:1 Repulsion. IntellectualArchive, v.8(3), P. 17. http://intellectualarchive.com/?link=item\&id=2164.

[49] Hodge, J. C. 2019g. Magnetic field evolves to gravity field:2 particles. IntellectualArchive, v.8(3), $\begin{array}{lll}\text { P. } & 30 .\end{array}$ http://intellectualarchive.com/?link=item\&id=2170.

[50] Hodge, J. C. 2019h. Magnetic field evolves to gravity field:3 Electromagnetics. IntellectualArchive, v. 8(3), P. 23. http://intellectualarchive.com/?link=item\&id=2193.

[51] Hodge, J. C. 2019i. Magnetic field evolves to gravity field:4 Atomic structure. IntellectualArchive, v. 8(4), P. 30. http://intellectualarchive.com/?link=item\&id=2199.

[52] Hodge, J. C. 2019j. Magnetic field evolves to gravity field:5 Final. IntellectualArchive, v.8(4), $\quad$ P. 37. http://intellectualarchive.com/?link=item\&id=2233.

[53] Hodge, J. C. 2020c. Magnetic field causes the strong and weak nuclear forces and is the GUT force. IntellectualArchive, v.9(3). http://intellectualarchive.com/?link=item\&id=2366. 